\title{
Diagnostic Yield of Emergency Department Arch-to-Vertex CT Angiography in Patients with Suspected Acute Stroke
}

\author{
A.R. Deipolyi, L.M. Hamberg, R.G. Gonzaléz, J.A. Hirsch, and G.J. Hunter
}

\begin{abstract}
BACKGROUND AND PURPOSE: Our aim was to investigate how often relevant diagnostic findings in an arch-to-vertex CTA scan, obtained specifically as part of the acute stroke CT protocol, are located in the head, neck, and upper chest regions.
\end{abstract}

MATERIALS AND METHODS: Radiology reports were reviewed in 302 consecutive patients (170 men, 132 women; median ages, 66 and 73 years, respectively) who underwent emergency department investigation of suspected acute stroke between January and July 2010. Diagnostic CTA findings relevant to patient management were recorded for the head, neck, and chest regions individually. Additionally, the contributions to the total CTA scan effective dose were estimated from each of the 3 anatomic regions by using the ImPACT CT Dose Calculator.

RESULTS: Of the 302 patients, 161 (54\%) had relevant diagnostic findings in the head; 94 (31\%), in the neck; and 4 (1\%), in the chest. The estimated contributions to the total CTA scan dose from each body region, head, neck, and upper chest, were $14 \pm 2 \%, 33 \pm 5 \%$, and $53 \pm$ $6 \%$, respectively.

CONCLUSIONS: Most clinically relevant findings are in the head and neck, supporting inclusion of these regions in arch-to-vertex CTA performed specifically in patients with acute stroke in the emergency department. Further studies are required to investigate extending the scan to the upper chest because only $1 \%$ of patients in our study had clinically relevant findings in the mediastinum, yet half the CTA effective dose was due to scanning in this region.

$\mathbf{I}_{\mathrm{m}}^{\mathrm{n}}$ $n$ the evaluation of patients presenting to the emergency department within 4.5 hours of the onset of acute stroke or strokelike symptoms, emphasis is placed on the immediate identification of inclusion and exclusion criteria for the administration of intravenous thrombolytic therapy. ${ }^{1-6}$ Subsequent investigation of risk factors for stroke is an elective process and typically does not begin until after the acute event has passed. ${ }^{7-9}$

With improvement in CT technology, the limitations on scan coverage of neurovascular CTA have relaxed, and single-pass imaging from the aortic arch to the skull vertex has become commonplace. ${ }^{10-15}$ Nevertheless, we have found no studies that evaluated benefit to the patient from this practice. Furthermore, current guidelines for the emergency management of patients

Received April 23, 2014; accepted after revision August 5.

From the Department of Radiology (A.R.D., R.G.G., J.A.H., G.J.H.), Massachusetts General Hospital, Boston, Massachusetts; and Department of Radiology (L.M.H.), Brigham and Women's Hospital, Boston, Massachusetts.

Please address correspondence to George J. Hunter, MD, PhD, Department of Radiology, Massachusetts General Hospital, 55 Fruit St, Boston, MA 02114; e-mail: gjhunter@partners.org

http://dx.doi.org/10.3174/ajnr.A4112 with acute stroke remain focused on noncontrast head CT and CTA evaluation only of the intracranial circulation. ${ }^{1,16-17}$

Our primary purpose was to investigate the incidence of diagnostically relevant findings reported in the head, neck, and chest components of an arch-to-vertex CTA performed in the emergency department as part of the immediate evaluation and triage of acute stroke. A secondary aim was to estimate the individual contributions to the total effective dose in these regions.

\section{MATERIALS AND METHODS \\ Patient Population and Clinical Findings}

This retrospective study was approved by our institutional review body, waiving consent in accordance with the Health Insurance Portability and Accountability Act. Included in the study were adult patients with signs and symptoms of acute stroke who presented to the emergency department between January 1 and July 31, 2010, and who underwent acute stroke CT imaging as part of their emergency diagnostic evaluation. There were no exclusion criteria. From the electronic medical record, age, weight, sex, NIHSS score at the time of presentation in the 
emergency department, and the treatment received were recorded for each patient.

All patients were included in the determination of the diagnostic information from individual regions of the CTA. The radiology reports were reviewed, and the presence and number of relevant diagnostic findings in the head, neck, and chest portions of the CTA scan were identified. Relevant diagnostic findings were defined as follows: 1) in the head, any vascular pathology affecting the intracranial circulation, for example, $>50 \%$ vessel stenosis, occlusion or partial occlusion of major vessels, loss of gray-white differentiation in a vascular territory, aneurysm, tumor, or hemorrhage; 2 ) in the neck, $>50 \%$ stenosis of the common or internal carotid arteries, $>70 \%$ stenosis of the vertebral arteries, or dissection of any vessel; and 3 ) in the chest, thrombus, dissection, or aneurysm of the aorta $>5 \mathrm{~cm}$. If there was uncertainty about the meaning of a report, joint review of both the report and the images was undertaken to resolve it. For the purposes of stenosis categorization, NASCET criteria were used. ${ }^{18}$

\section{CTA Imaging}

CTA scanning was performed as part of the acute stroke CT protocol on either a 64- or 16-section multidetector row CT scanner (LightSpeed VCT and LightSpeed Pro16; GE Healthcare, Milwaukee, Wisconsin). For the CTA, a single axial scan was acquired through the aortic root and a region of interest was placed in the ascending aorta for use with SmartPrep software (GE Healthcare), a manufacturer-specific bolus-tracking method that allows consistent timing of CTA imaging with respect to an individual patient's contrast hemodynamics. Contrast material (iopamidol, Isovue 370; Bracco Diagnostics, Princeton, New Jersey) was injected in 2 phases: first, $60 \mathrm{~mL}$ at $4 \mathrm{~mL} / \mathrm{s}$, immediately followed by $15 \mathrm{~mL}$ at $2 \mathrm{~mL} / \mathrm{s}$, followed by a $40-\mathrm{mL}$ saline chase. The change in Hounsfield unit number in the aortic region of interest was monitored during injection; CTA imaging was triggered $10 \mathrm{sec}-$ onds after this change exceeded 75 Hounsfield units. On the 64section scanner, the scanning parameters for CTA were the following: $120 \mathrm{kV}$ (peak) x-ray tube voltage, automatic tube-current modulation, $32-\mathrm{cm}$ scan FOV, $64 \times 0.625 \mathrm{~mm}$ detector configuration, 0.5 -second gantry rotation time, a 0.52 pitch factor, and 40-mm beam width. On the 16-section scanner, the CTA scanning parameters were the following: either 120 or $140 \mathrm{kVp}$ x-ray tube voltage, automatic tube current modulation, $25-\mathrm{cm}$ scan FOV, $16 \times 0.625 \mathrm{~mm}$ detector configuration, 0.5 -second gantry rotation time, 0.94 pitch factor, and $10-\mathrm{mm}$ beam width. For all protocols, $1.25-\mathrm{mm}$ images were reconstructed at $0.625-\mathrm{mm}$ intervals by using a $512 \times 512$ image matrix and the manufacturer's standard reconstruction kernel.

\section{Effective Dose Estimation}

Effective dose contributions for each protocol were estimated by using the ImPACT CT Patient Dosimetry Calculator (ImPACT, London, United Kingdom). ${ }^{19-20}$ This software uses a mathematic, anthropomorphic phantom to represent an average-sized, $70-\mathrm{kg}$ reference adult. If used for effective dose estimation in smaller patients, the software underestimates the

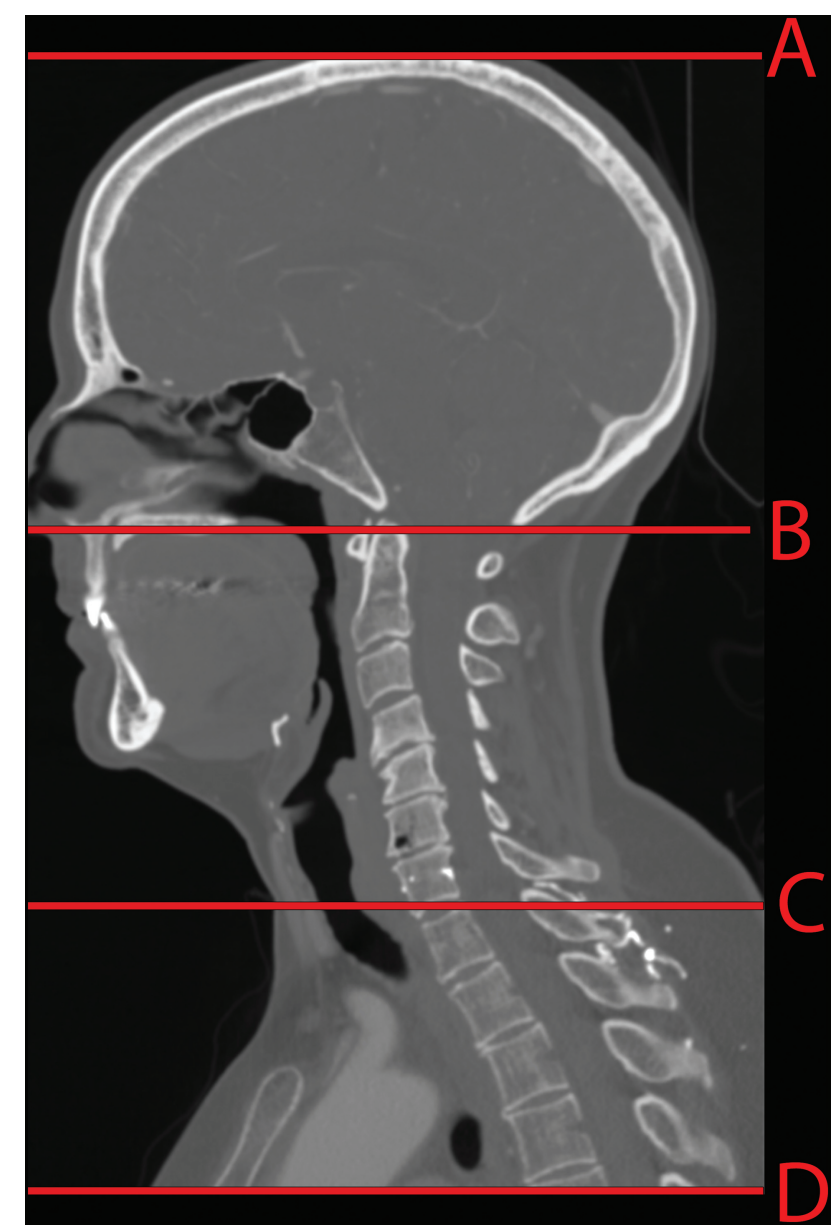

FIGURE. Template for the anatomic landmarks used to determine the head, neck, and chest regions. The red horizontal lines on the sagittal reformat demonstrate the location of the 4 landmarks used: the skull vertex $(A)$, the top of the $\mathrm{Cl}$ arch $(B)$, the bottom of the $\mathrm{C7}$ vertebra $(C)$, and the most inferior section scanned $(D)$. The head region was defined as between $A$ and $B$; the neck region, between $B$ and $C$; and the chest region, between $C$ and $D$.

effective dose, and in larger patients, it overestimates it. Thus, only those patients in our cohort with weights within $10 \mathrm{~kg}$ of the reference adult $(60-80 \mathrm{~kg})$ were included in the effective dose analysis.

Because all 3 CTA protocols had the tube current modulation option turned on, an average milliampere value used for the head, neck, and upper chest region was determined for the patients studied. This was facilitated by writing custom software (Visual Studio 2010; Microsoft, Redmond, Washington) that obtained the milliampere values for each section within a CTA series, together with their corresponding section location coordinates from the DICOM headers. From these data, a milliampere value was individually determined for each body region for all patients.

The body regions were determined by using anatomic landmarks. The head region was counted from the skull vertex to the top of the $\mathrm{C} 1$ ring; the neck region was taken from the top of the dens to the bottom of the $\mathrm{C} 7$ vertebra; and the chest region, from the bottom of $\mathrm{C} 7$ to the lowest level scanned (Figure). The start and end locations for each body region were recorded on our institutional PACS system (Impax 5.3; Agfa-Gevaert, Mortsel, 
Belgium) as the section location coordinates marking the relevant body region boundaries.

Descriptive and comparative statistics and significance testing (unpaired Student $t$ test) were performed by using Excel 2010 (Microsoft).

\section{RESULTS}

Of the 302 patients identified in the study period, there were 170 men (mean age, 65 years; median age, 66 years; range, 19-90 years) and 132 women (mean age, 69 years; median age, 73 years; range, 23-100 years). Weight measurements were available in 267 patients $(88 \%, 267 / 302)$ with a mean of $82 \pm 19 \mathrm{~kg}$. There were 111 patients weighing $60-80 \mathrm{~kg}(42 \%, 111 / 267)$, with a mean of $71 \pm 6 \mathrm{~kg}$. Data for these patients were used for estimation of effective dose contributions.

In all patients, the average NIHSS score at the time of presentation to the emergency department was 7.7 (range, 0-26). Ninety-three patients $(31 \%, 93 / 302)$ were eligible for IV-tPA. Of these, $63 \%$ (59/93) received IV-tPA; 37\% did not because they fell outside the time window for treatment $(<4.5$ hours), because their lesion was too large (more than one-third of the MCA territory), or there were medical contraindications such as recent trauma, systemic bleeding, or surgery; $6.6 \%$ of patients (20/302) proceeded to intra-arterial therapy. The mean NIHSS score in the intra-arterially treated group was 12.4 , which was significantly higher than that in the remaining patients who had an average scale value of $7.3(P<.01)$.

In the head portion of the CTA, $53 \%$ of patients $(161 / 302)$ had significant vascular-related pathology. In $78 \%$ of these patients (129/161), the reported findings were directly relevant to the patient's ischemic stroke symptoms, with vessel cutoff in the territory of the patient's infarct.

In the neck portion of the CTA, relevant vascular pathology was found in $31 \%$ of patients (94/302). Of these, $64 \%$ (60/94) had at least moderate internal carotid artery stenosis, 30\% (28/94) had significant vertebral artery stenosis, 6\% (6/94) had both vertebral and internal carotid arterial stenosis, and 4\% (4/94) had internal carotid or vertebral artery dissections.

In the upper chest, relevant vascular pathology was found in $1.3 \%$ of patients (4/302); this consisted of extensive aortic plaque associated with moderate or severe stenosis of a common carotid origin in all 4 cases.

The subset of 111 average-sized patients, for whom the body region contributions to the total effective dose were estimated, demonstrated a $14 \pm 2 \%, 33 \pm 5 \%$, and $53 \pm 6 \%$ contribution to the total effective dose from the head, neck, and chest regions, respectively.

\section{DISCUSSION}

We have found that a very small minority of findings on neurovascular CTA, performed expressly for the purposes of triage of patients with acute stroke symptoms, are in the chest. While some neurointerventional experts argue for the routine inclusion of the upper chest in an emergency department CTA, it is unclear how often 3D road maps from the scan are actually available before intra-arterial intervention. Increasing public awareness of potential radiation risks from medical $\mathrm{x}$-rays has resulted in renewed scrutiny of the radiation dose burden from CT scanning. ${ }^{21}$ We found that approximately half of the effective dose from arch-tovertex CTA is due to the chest portion of the scan because of the high radiosensitivity of the lungs. ${ }^{22}$ On the basis of our observations, the chest portion of the CTA does not appear to contribute meaningfully to the management or triage of patients with acute stroke; thus, an approximately 50\% reduction in effective dose could be achieved simply by limiting CTA to the vasculature above the shoulders, without compromising patient care.

The present study is limited by its retrospective nature because only the radiology report was used to determine the presence of relevant findings in the 3 regions of the CTA. In the acute setting, interpretation of the images by a neuroradiologist, as they appear on the scanner console, happens in real- or near-real-time, meeting the recommendations of the American Heart Association that stipulate interpretation of imaging studies within 45 minutes of patient arrival in the emergency department. ${ }^{1}$ Discussion among neuroradiology, neurointerventional, and neurology personnel at that time will likely have a substantial impact on the chosen management pathway, yet it may not have been fully represented in the final radiology report and thus may have been unavailable for the present analysis. Thus, we may under-represent the actual findings used at the time of treatment. Addressing this issue and the issues of road-mapping before intra-arterial therapy and the management value of the intrathoracic component of the CTA would require prospective trials with appropriate power to answer these questions definitively. Another potential limitation is how well our study cohort represents the larger population of patients with acute stroke. Given the low number of relevant positive findings in the chest in 302 patients, we believe it is unlikely that substantially different results would be found in a larger study group.

\section{CONCLUSIONS}

In summary, we found that CT angiography of the head, neck, and upper chest performed in the emergency department, specifically to manage patients with acute stroke symptoms, provided diagnostically useful, potentially management-altering information only from the head and neck components of the scan. No information relevant to acute management of such patients was forthcoming from the chest portion of the CTA, yet this component accounted for approximately $50 \%$ of the effective dose of the CTA scan. Further evidence-based studies are needed to more fully evaluate these findings.

Disclosures: Joshua A. Hirsch—UNRELATED: Consultancy: CareFusion, Comments: Received fees related to vertebral augmentation; Stock/Stock Options: stroke development company.

\section{REFERENCES}

1. Jauch EC, Saver JL, Adams HP, et al. Guidelines for the early management of adults with ischemic stroke: a guideline for healthcare professionals from the American Heart Association/American Stroke Association. Stroke 2013;44:870-947

2. Del Zoppo GJ, Saver JL, Jauch EC, et al. Expansion of the time window for treatment of acute ischemic stroke with intravenous tissue plasminogen activator: a science advisory from the American Heart Association/American Stroke Association. Stroke 2009;40:2945-48

AJNR Am J Neuroradiol 36:265-68 Feb 2015 www.ajnr.org 267 
3. Wechsler LR. Intravenous thrombolytic therapy for acute ischemic stroke. N Engl J Med 2011;364:2138-46

4. Wardlaw JM, Murray V, Berge E, et al. Recombinant tissue plasminogen activator for acute ischaemic stroke: an updated systematic review and meta-analysis. Lancet 2012;379:2364-72

5. Adair G, Grant J, Pandhi N, et al. Clinical inquires: when should patients with stroke receive thrombolytics? J Fam Pract 2004;53:656, $658-59$

6. Hacke W, Kaste M, Bluhmki E, et al. Thrombolysis with alteplase 3 to 4.5 hours after acute ischemic stroke. $N$ Engl J Med 2008; 359:1317-29

7. Morris JG, Duffis EJ, Fisher M. Cardiac workup of ischemic stroke: can we improve our diagnostic yield? Stroke 2009;40:2893-98

8. Chung JH, Mitsumori LM, Ordovas KG, et al. Heart as a source of stroke: imaging evaluation with computed tomography. J Thorac Imaging 2012;27:W52-60

9. Boussel L, Cakmak S, Wintermark M, et al. Ischemic stroke: etiologic work-up with multidetector CT of the heart and extra- and intracranial arteries. Radiology 2011;258:206-12

10. Shrier DA, Tanaka H, Numaguchi $Y$, et al. CT angiography in the evaluation of acute stroke. AJNR Am J Neuroradiol 1997;18:1011-20

11. Knauth M, von Kummer R, Jansen $\mathrm{O}$, et al. Potential of CT angiography in acute ischemic stroke. AJNR Am J Neuroradiol 1997; 18:1001-10

12. Hunter GJ, Hamberg LM, Ponzo JA, et al. Assessment of cerebral perfusion and arterial anatomy in hyperacute stroke with threedimensional functional CT: early clinical results. AJNR Am J Neuroradiol 1998;19:29-37

13. Smith WS, Roberts HC, Chuang NA, et al. Safety and feasibility of a CT protocol for acute stroke: combined CT, CT angiography, and
CT perfusion imaging in 53 consecutive patients. AJNR Am J Neuroradiol 2003;24:688-90

14. Latchaw RE, Alberts MJ, Lev MH, et al. Recommendations for imaging of acute ischemic stroke: a scientific statement from the American Heart Association. Stroke 2009;40:3646-78

15. Leiva-Salinas $C$, Wintermark M. Imaging of acute ischemic stroke. Neuroimaging Clin N Am 2010;20:455-68

16. Gentile NT, Cernetich J, Kanamalla US, et al. Expedited computed tomography perfusion and angiography in acute ischemic stroke: a feasibility study. J Emerg Med 2012;43:308-15

17. Kidwell CS, Wintermark M. The role of CT and MRI in the emergency evaluation of persons with suspected stroke. Curr Neurol Neurosci Rep 2010;10:21-28

18. Chang YJ, Golby AJ, Albers GW. Detection of carotid stenosis: from NASCET results to clinical practice. Stroke 1995;26:1325-28

19. Jones DG, Shrimpton PC. Survey of CT Practice in the UK. Part 3: Normalized Organ Doses Calculated using Monte Carlo Techniques. National Radiological Protection Board-250. London: Her Majesty's Stationery Office, 1991

20. Shrimpton PC, Jones DG, Hillier MC, et al. Survey of CT Practice in the UK. Part 2: Dosimetric Aspects. National Radiological Protection Board-R249. London: Her Majesty's Stationery Office; 1991

21. Boone JM, Hendee WR, McNitt-Gray MF, et al. Radiation exposure from CT scans: how to close our knowledge gaps, monitor and safeguard exposure: proceedings and recommendations of the Radiation Dose Summit, sponsored by National Radiological Protection Board, February 24-25, 2011. Radiology 2012;265:544-54

22. International Commission on Radiological Protection. The 2007 Recommendations of the International Commission on Radiological Protection. Stockholm: International Commission on Radiological Protection. ICRP Publication 103, Ann. ICRP 37 (2-4), 2007 